\title{
Mapping neuronal activation and the influence of adrenergic signaling during contextual memory retrieval
}

\author{
Wei-Ping Zhang, ${ }^{1,3}$ John F. Guzowski, ${ }^{2}$ and Steven A. Thomas ${ }^{1,4}$ \\ ${ }^{1}$ Department of Pharmacology, University of Pennsylvania, Philadelphia, Pennsylvania 19104-6084, USA; ${ }^{2}$ Department of \\ Neurosciences, University of New Mexico, Albuquerque, New Mexico 87131-5223, USA
}

\begin{abstract}
We recently described a critical role for adrenergic signaling in the hippocampus during contextual and spatial memory retrieval. To determine which neurons are activated by contextual memory retrieval and its sequelae in the presence and absence of adrenergic signaling, transcriptional imaging for the immediate-early gene Arc was used in control and mutant mice lacking norepinephrine and epinephrine. This imaging approach permits the identification of neuronal genomic activation specific to one of two behavioral epochs in the same animal. Analysis revealed several brain regions that were more greatly activated by re-exposure to a salient versus neutral context $1 \mathrm{~d}$ after training in control mice (e.g., hippocampal CA3 and CAl, the amygdala, the dorsolateral caudate/putamen, the primary motor cortex, and parts of the rhinal cortices). In mice lacking norepinephrine and epinephrine, many of these regions exhibited significantly reduced activation (e.g., hippocampal CAl), while other regions did not (e.g., hippocampal CA3). In consideration with previous results, the current findings suggest a hypothesis in which adrenergic signaling may be critical for the transfer of retrieved contextual information from CA3 to CA1, where it would be compared to online sensory information coming directly from the cortex.
\end{abstract}

One goal for understanding memory is to define the neurons activated during memory storage and retrieval. Electrophysiologic recordings and brain lesions have been mainstays in this pursuit. More recently, imaging techniques have been used. These include functional brain imaging studies in humans using positron emission tomography (PET) and functional magnetic resonance imaging (fMRI), which are noninvasive and permit repeated measurements; however, they cannot resolve activity at the cellular level (Schacter and Wagner 1999; Mayes and Montaldi 2001). For this, induction of immediate-early gene (IEG) expression ("genomic activation") has been used in animals, offering high sensitivity and cellular resolution that can be analyzed throughout the brain (Clayton 2000). A disadvantage, however, is that each animal is assessed under a single condition.

A technique that overcomes this disadvantage uses fluorescence in situ hybridization (FISH) to the IEG Arc. Arc (Lyford et al. 1995), also termed Arg3.1 (Link et al. 1995), is an activityregulated, cytoskeleton-associated protein implicated in synaptic plasticity and memory (Steward et al. 1998; Guzowski et al. 2000). Basal expression of Arc is very low in most neurons. However, transcription is rapidly induced by neuronal activation, and FISH to Arc reveals two small, intense intranuclear foci within 2 min of activation (Guzowski et al. 1999). By 20 min the nuclear signal has disappeared and perinuclear cytoplasmic labeling is transiently observed before disappearing due to dendritic transport of Arc mRNA (Steward et al. 1998). These properties allow one to determine the recent activation history of a neuron at two distinct times, referred to as cellular compartment analysis of

\footnotetext{
${ }^{3}$ Present address: Department of Pharmacology, Zhejiang University School of Medicine, Zhejiang, Hangzhou, 310031, P.R. China. ${ }^{4}$ Corresponding author.

E-mail thomas@pharm.med.upenn.edu; fax (215) 573-2236. Article and publication are at http://www.learnmem.org/cgi/doi/10.1101/ Im.90005.
}

temporal activity by $\underline{\text { FISH }}$ (catFISH) (Guzowski et al. 1999, 2001). Arc catFISH has been used to examine activation of hippocampal pyramidal neurons in identical and distinct contexts (Guzowski et al. 1999), generating results supported by electrophysiologic recordings (Kubie and Ranck 1983; Thompson and Best 1990; Wilson and McNaughton 1993).

One goal of this study was to determine whether Arc catFISH would identify neurons uniquely activated as a result of exposure to a salient versus neutral context (the former resulting in the retrieval of a contextual fear memory). If it could, activation of these neurons would likely reflect a variety of processes related to contextual fear, including retrieval of the contextual fear memory, increased arousal or fear following retrieval, or conditioned responses generated by fear, such as freezing. Our analysis included the hippocampus for several reasons. First, induction of Arc occurs readily in hippocampal pyramidal neurons following a change in context (Guzowski et al. 1999). Second, the hippocampus is critical for contextual memory storage and discrimination of contexts during memory retrieval (Kim and Fanselow 1992; Kim et al. 1992; Phillips and LeDoux 1992; Holt and Maren 1999). Third, antisense knock-down of Arc expression in the hippocampus reduces the expression of long-term potentiation and spatial memory (Guzowski et al. 2000). This is likely due to a role for $A r c$ in consolidation because short-term synaptic plasticity and short-term memory remain intact. Fourth, induction of Arc also occurs when animals are returned to a previously experienced context. This could be due to a role for Arc in habituation, reconsolidation, or consolidation of new memory, processes that likely require memory retrieval (Nader et al. 2000; Myers and Davis 2002). While induction of Arc is not necessary for memory retrieval, it seems likely that it may identify neurons genomically activated as a result of retrieval.

Besides the hippocampus, we examined additional brain regions, a number of which have not been studied using Arc catFISH before, to determine whether this technique could identify neurons in these regions uniquely activated following expo- 
sure to the salient versus neutral context. We anticipated that regions such as the amygdala, for example, might exhibit such labeling, given its critical role in fear and conditioned responding (Fanselow and LeDoux 1999).

A second goal was to determine whether activation of neurons specific to a salient context depends on adrenergic signaling. We recently demonstrated a critical and specific role for norepinephrine (NE) in contextual and spatial memory retrieval that is not due to a role in arousal, fear, or performance (Murchison et al. 2004). Those findings were initiated by the study of mice genetically altered to lack the endogenous ligands for the adrenergic receptors, $\mathrm{NE}$ and epinephrine (NE/E), via targeted disruption of the dopamine $\beta$-hydroxylase gene $(D b h)$ (Thomas et al. 1995, 1998). Intracerebral infusions indicated that $\beta_{1^{-}}$adrenergic receptor signaling in the hippocampus is necessary and sufficient for contextual memory retrieval mediated by NE. As a result, we anticipated that a subset of neurons identified by $A r c$ catFISH as being activated specifically during exposure to the salient context might not be activated in the absence of NE/E, and that at least some of those neurons would reside in the hippocampus, because of the role of NE in that structure for contextual memory retrieval. We also anticipated that additional brain regions, such as the amygdala, might exhibit reduced salient context-specific activation in the absence of NE/E because of differences in fear and conditioned responding.

\section{Results}

\section{Arc catFISH in mice}

We first examined the time course of Arc expression in our mice because previous studies were performed in rats. We used reexposure to the training apparatus $1 \mathrm{~d}$ after fear conditioning as the stimulus for induction of Arc to match our main experimental design (see below). Mice were sacrificed 5-35 min after reexposure. Figure 1 identifies the brain regions examined using Arc catFISH. At 5 min there were significant increases in nucleusspecific labeling that returned to baseline by $20 \mathrm{~min}$ in all brain regions examined except the dentate gyrus (DG, not shown) (Fig. 2). In rat, $\operatorname{Arc}$ transcription initiated by a single stimulus persists for hours in the DG, as compared to minutes in other excitatory cell populations of hippocampus and neocortex (Vazdarjanova et al. 2002), in agreement with the current findings in mice. In all other regions, cytoplasm-specific labeling peaked at $\sim 30 \mathrm{~min}$, and the percentages of labeled cells were equivalent to those for nucleus-specific labeling at $5 \mathrm{~min}$, suggesting that essentially all nucleus-positive neurons become nucleus-negative, cytoplasmpositive neurons between 5 and $30 \mathrm{~min}$. These observations are consistent with the time course of $A r c$ induction in rats (Guzowski et al. 1999), and indicate that Arc catFISH can be used in mice to analyze neuronal activation in many brain regions following context exposure.
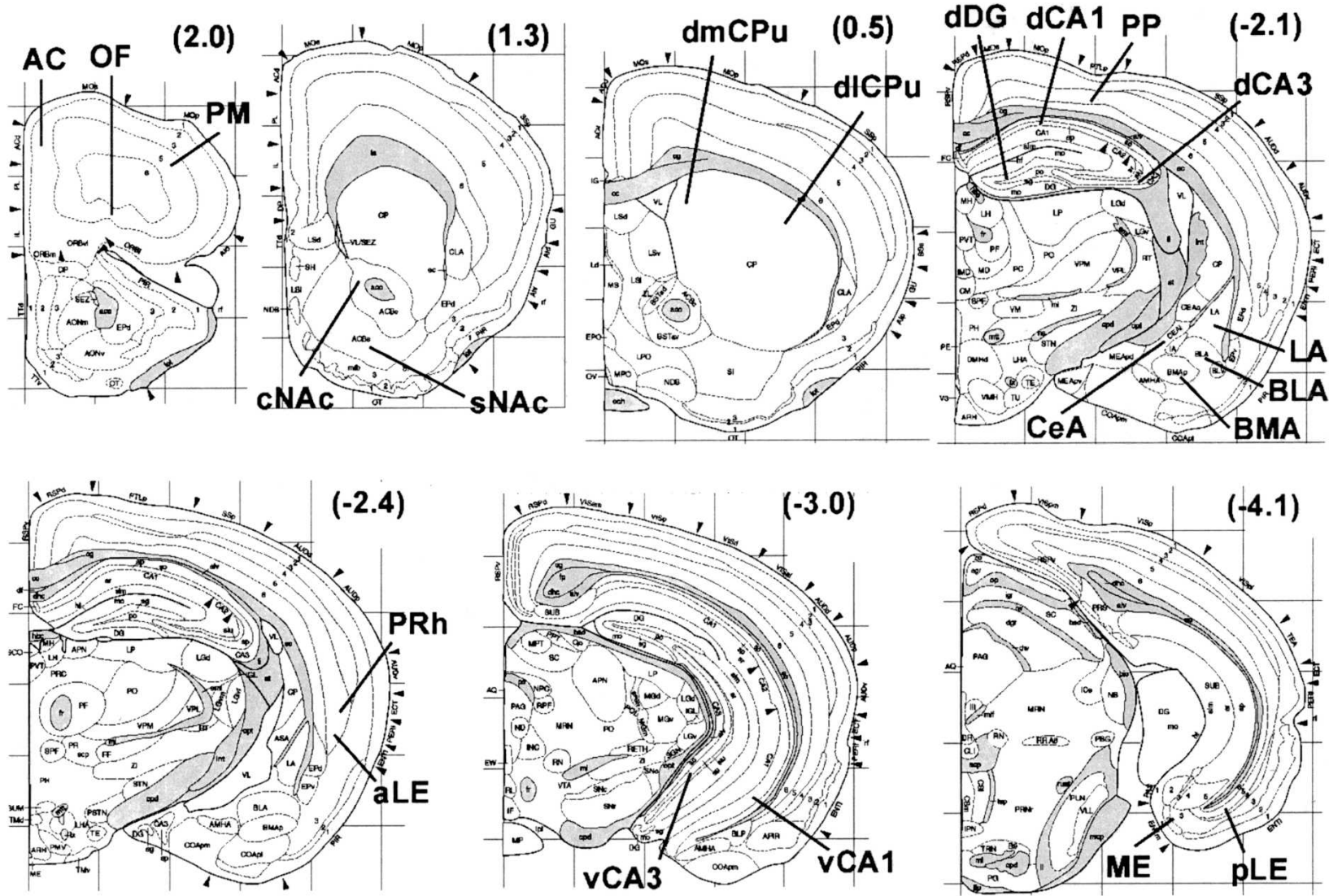

Figure 1. Coronal mouse brain diagrams with regions of analysis indicated. Numbers in parentheses are anterior-posterior distances from Bregma in millimeters. Abbreviations of regions-for hippocampus: dCA3 and dCA1 are dorsal CA3 and CA1; vCA3 and vCA1 are ventral CA3 and CA1; for amygdala: LA, BLA, BMA, and CeA are lateral, basolateral, basomedial, and central nuclei; for striatum: dICPu and dmCPu are dorsolateral and dorsomedial caudate/putamen; sNAc and cNAc are the shell and core regions of the nucleus accumbens; for cortex: ME, pLE, and aLE are medial, posterior lateral and anterior lateral entorhinal; PRh is perirhinal; PP is posterior parietal; OF is orbitofrontal; AC is anterior cingulate; PM is primary motor. Diagrams are reprinted with permission from Elsevier Science $\odot 2000$ (Hof et al. 2000). 

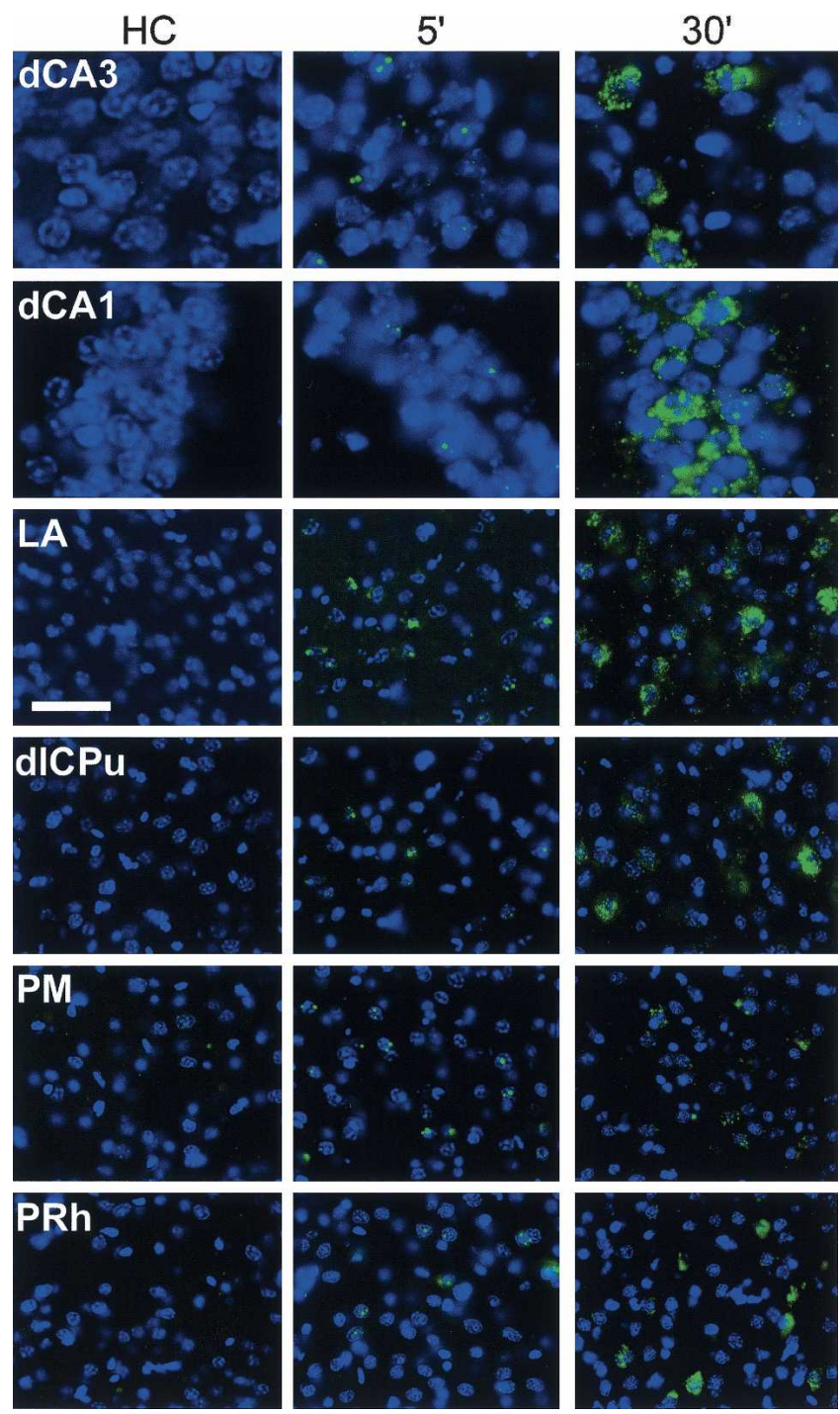

Figure 2. $\operatorname{Arc}$ expression before $(\mathrm{HC})$ and after (5 min and $30 \mathrm{~min}$ ) re-exposure to the training context. Sections are from mice that were sacrificed $1 \mathrm{~d}$ after training with shock. For $5 \mathrm{~min}$ and $30 \mathrm{~min}$, mice were returned to their home cage after exposure to context $S$ on Day 2 for 5 min and 30 min before sacrifice, respectively. Nuclei are blue and Arc RNA is green. Almost no labeling is present in HC. At 5 min, small intranuclear foci of labeled Arc RNA are present in some neurons in each brain region. At 30 min, perinuclear cytoplasmic labeling of $\operatorname{Arc}$ mRNA is present and intranuclear labeling is similar to that for HC. Regions are as per Figure 1. The scale bar is $40 \mu \mathrm{m}$ for all regions except dCA3 and dCA1, where it is $20 \mu \mathrm{m}$.

\section{Design of Arc catFISH}

To identify neurons activated during and following retrieval of contextual fear, paradigms using two contexts were used. Context $\mathrm{S}$ (shock or salient) was the training apparatus and context $\mathrm{N}$ (non-shock or neutral) was a distinct context not associated with shock. For the main two experimental groups, neuronal activation in the salient versus neutral context was assessed. Mice were exposed to both contexts on the training day, receiving shock in context $\mathrm{S}$ (Fig. 3A). These mice were then re-exposed to each context on the testing day $1 \mathrm{~d}$ later. One of these groups was exposed to context $\mathrm{S}$ then $\mathrm{N}$ (group $\mathrm{S} 1$ = context $\mathrm{S}$ first), the other to context $\mathrm{N}$ then $\mathrm{S}$ (group $\mathrm{S} 2$ = context $\mathrm{S}$ second) to control for order effects. For a third group of mice, neuronal activation in two neutral contexts was assessed. Mice were exposed to context
$\mathrm{N}$ then $\mathrm{S}$ during training without receiving shock, and the next day were exposed to context $\mathrm{S}$ then $\mathrm{N}$ (group NS = no shock during training). A fourth group of mice was used to assess basal levels of Arc expression following fear conditioning. These mice were exposed to both contexts on the training day, receiving shock in context $\mathrm{S}$. They were then sacrificed from their home cage the next day (group $\mathrm{HC}=$ home cage). Using these paradigms, fear conditioning (assessed by freezing) was apparent in groups S1 and S2 but not NS, as expected (Fig. 3B). The $D b h^{-1-}$ mice froze significantly less than controls, consistent with an impairment in contextual memory retrieval (Murchison et al. 2004). Groups S1 and S2 were combined to create group SX because significant differences within genotype were absent.

\section{Total Arc expression: Training history} and adrenergic signaling

To examine how Arc expression varies between regions, treatment groups, and genotypes, total Arc labeling was analyzed. Importantly, total percentages of Arc expression (nuclear plus cytoplasmic plus double-labeled/total cells analyzed) did not differ by genotype in any brain region except for the primary motor cortex, where Arc was reduced in the $\mathrm{Dbh}^{-1-}$ mice because of a large difference in salient context-specific Arc expression (see below). These data indicate that there is not a general impairment in induction of $A r c$ in the absence of NE/E. Two common patterns of $A r c$ induction were observed between treatment groups. One pattern identified brain regions that were primarily contextsensitive. In these regions there were increases in total labeling in the non-shock group compared to the home-cage group, without further significant increases in the shock group compared to the non-shock group (Fig. 4). This pattern was observed in the hippocampus, core of the nucleus accumbens, dorsomedial caudate/ putamen, and the perirhinal, posterior parietal, and orbitofrontal cortices. The other pattern identified regions that were sensitive to training condition. It was similar to the first pattern except that there were significant increases in total labeling in the shock group compared to the non-shock group. This pattern was observed in the amygdala, shell of the nucleus accumbens, dorsolateral caudate/putamen, and the entorhinal, anterior cingulate, and primary motor cortices. Unlike all other regions, in the DG

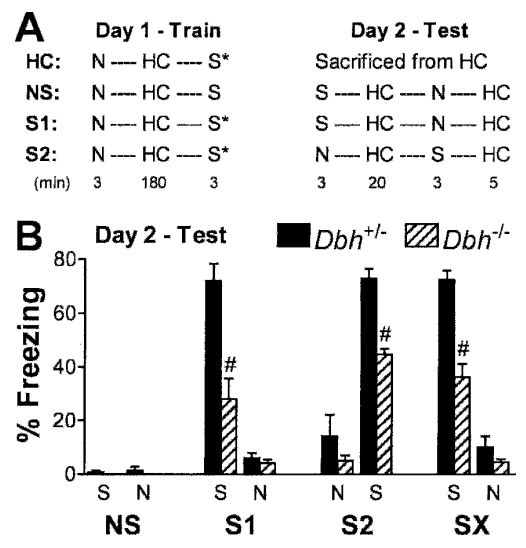

Figure 3. Protocol for Arc catFISH and freezing behavior of mice. (A) The order and duration of context exposures are shown. All groups were trained with exposure to context $\mathrm{N}$ followed by a 3-h epoch in their home cage $(\mathrm{HC})$ and then exposure to context S. All mice received shock ${ }^{*}$ ) on Day 1 in context $S$ except group NS. Mice were tested the next day as shown without any shock. NS, S1, and S2 groups were sacrificed after the second $\mathrm{HC}$ epoch on Day 2. (B) Freezing during testing on Day 2. Group SX represents the combined data from groups S1 and S2 (thus $n=8$ per genotype). For all figures, levels of significance are indicated as follows: $\left(^{*}\right) P<0.05 ;(\wedge) P<0.01$; (\#) $P<0.001$. 

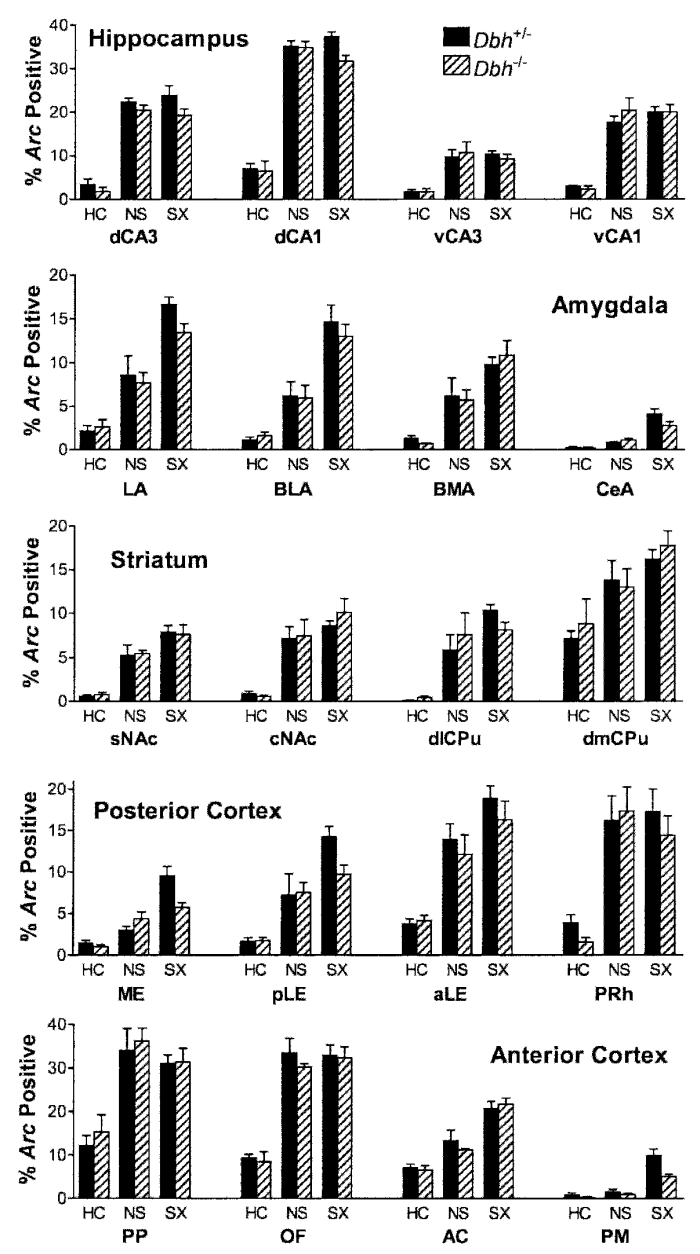

Figure 4. Total Arc labeling by brain region, treatment group, and genotype. Shown are the combined percentages of cells labeled for Arc either in the nucleus, the cytoplasm, or both. Regions are as per Figure 1 and groups are as per Figure 3. The main effect of treatment was significant for all regions $(P<0.001)$. There were no significant main effects of genotype except for PM cortex $(P=0.03)$ and no significant interactions between treatment and genotype. When collapsed by genotype, labeling in NS was significantly higher $(P<0.01)$ than $\mathrm{HC}$ for all regions except PM. In addition, SX was significantly higher $(P<0.05)$ than NS for all regions in the amygdala and entorhinal cortex, as well as the AC and PM cortex.

there was an increase only in double-positive labeling with context exposure (Fig. 5A). This was anticipated in part from the extended time course of nuclear labeling in the DG. These same overall labeling patterns described above were also apparent when labeling was parceled into the three categories that indicate when each neuron was activated: cytoplasmic $(\mathrm{Cy}$, specific for the first epoch), nuclear (Nu, specific for the second epoch), and double-labeled (Do, common to both epochs) (Fig. 6; data not shown for regions outside the hippocampus). Labeling was parceled in this manner so that neurons activated during each behavioral epoch could be quantified and compared (see below).

\section{Identification of salient context-specific labeling using Arc catFISH}

To identify brain regions that contained neurons activated during and following contextual memory retrieval, we examined whether the shock groups S1 and S2 would exhibit significantly more uniquely activated neurons in context $\mathrm{S}$ versus $\mathrm{N}$. There- fore, labeling resulting from context $\mathrm{N}$ was subtracted from that for context $\mathrm{S}$ for each mouse ( $\mathrm{S}-\mathrm{N}=$ residual labeling). Because double labeling (Do) is common to both contexts, $\mathrm{S}-\mathrm{N}$ is represented by the difference between cytoplasm- and nucleusspecific labeling (Cy - Nu for group S1; $\mathrm{Nu}$ - Cy for group S2). We then tested whether the residual labeling was significantly different from zero. For the non-shock group, values were expected to be not significantly different from zero (i.e., $\mathrm{Cy}=\mathrm{Nu}$ ) in order to satisfy the basic premise of catFISH. Importantly, this was confirmed for all brain regions examined in both genotypes except the DG (Figs. 5-7). The different outcome for the DG was expected based on the extended time course of Arc labeling in the granule cells. For the shock groups, we asked whether residual Arc labeling might be significantly greater than zero in any brain region. If it were, this would indicate that Arc catFISH could be used to identify regions containing neurons uniquely activated as a result of contextual fear memory retrieval or its sequelae, such as increased arousal, fear, or conditioned fear responses like freezing. To control for possible order effects on induction or detection of Arc, groups S1 and S2 were combined to create group SX.

\section{Salient context-specific labeling in the hippocampus}

We first examined the hippocampus for significant salient context-specific labeling for Arc in control mice because lesion and reversible inactivation studies have demonstrated an important role for the hippocampus in contextual memory storage and retrieval (Kim and Fanselow 1992; Kim et al. 1992; Phillips and LeDoux 1992; Holt and Maren 1999). We also examined the hippocampus because adrenergic signaling in this region is necessary for contextual fear memory retrieval $1 \mathrm{~d}$ after training (Murchison et al. 2004). However, it is not known in which subfield(s) of the hippocampus activation might be affected during memory retrieval in the absence of NE/E.

In the dorsal hippocampus, we found significant salient context-specific labeling in dCA3 and dCA1 in control mice, which was apparent when inspecting percentages of cytoplasmand nucleus-specific labeling in the shock groups (S1 and S2; left part of Fig. 6), and was shown quantitatively by the residual
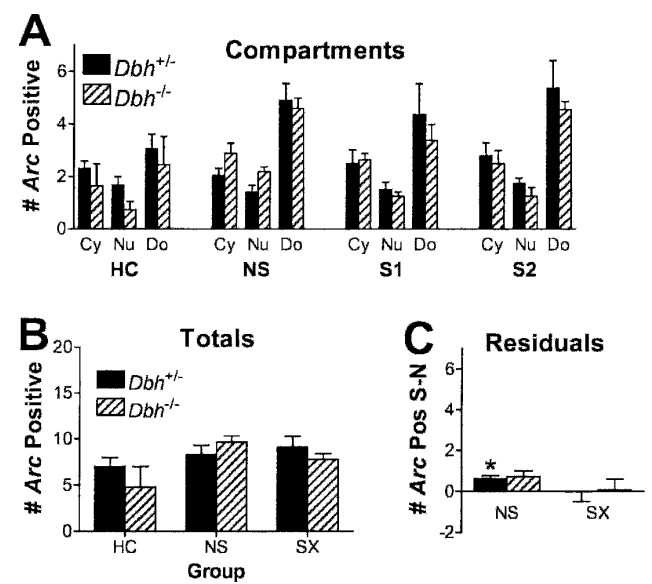

Figure 5. Compartment analysis of Arc labeling in the dentate gyrus. Data are presented as per Figures 4 and 6, except that instead of reporting the percentage of cells labeled, the number of labeled cells per section is given owing to the sparse labeling in the DG. $(A)$ The main effect of compartment labeling was significant for all four groups. (B) The main effect of treatment was significant. (C) For control mice, there was significant bias in the NS group that reflected the greater amount of cytoplasmic to nuclear labeling in the DG. No significant salient contextspecific labeling was observed.

\section{Learning \& Memory} www.learnmem.org 

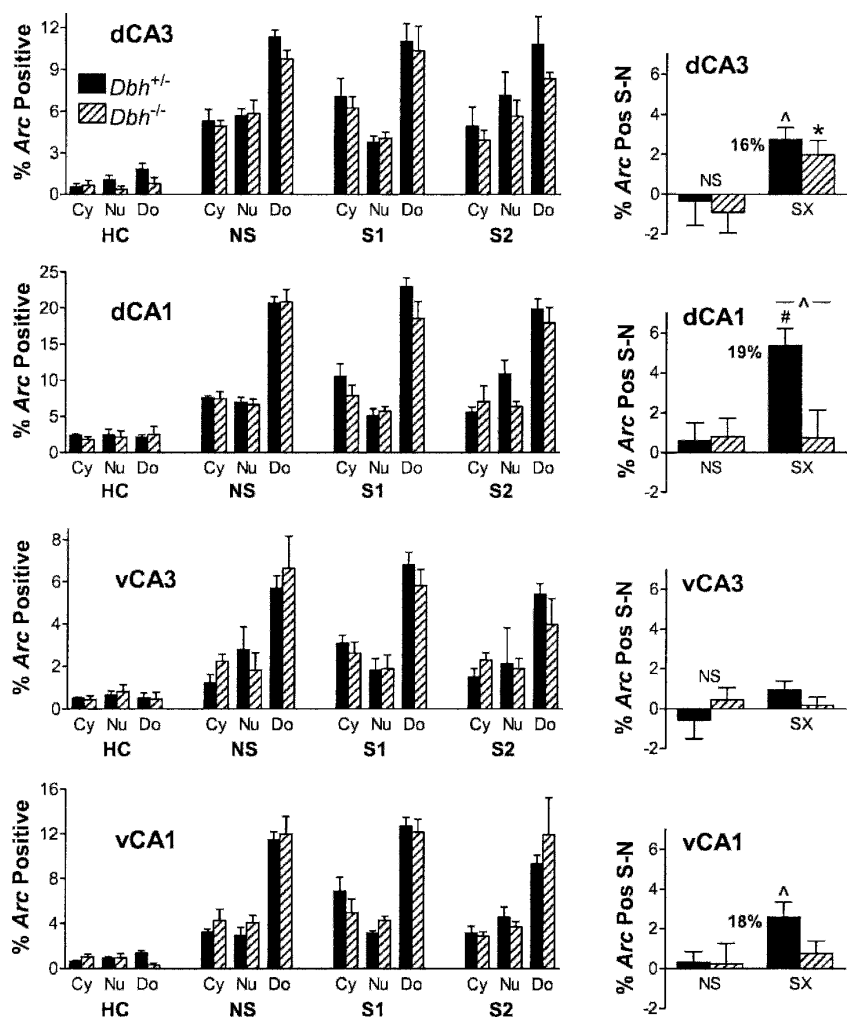

Figure 6. Compartment analysis of Arc labeling and identification of salient context-specific labeling in the hippocampus. On the left are the percentages of cells labeled either in the cytoplasm (Cy, unique labeling for the first epoch), the nucleus ( $\mathrm{Nu}$, unique labeling for the second epoch), or both (Do, double, common to both epochs) for each treatment. Only the main effect of Arc labeling by compartment was significant, and this was true for groups NS, S1 and S2 (but not HC) for each region $(P<0.01)$. On the right are the mean residual labeling percentages after subtracting the values for context $\mathrm{N}$ from context $\mathrm{S}$ for each mouse, as a percentage of all cells analyzed, including unlabeled neurons. Significant residual (salient context-specific) labeling was observed in dCA3, dCA1, and vCA1 for the control mice and dCA3 for the $D b h^{-1-}$ mice. There was a significant difference in residual labeling between genotypes for dCA1. Numbers next to $D b h^{+/-}$SX bars indicate the percent increase in $\operatorname{Arc}$ labeling in context $S$ relative to context $N$ when significant residual labeling is present.

labeling percentages (right part of Fig. 6). Note that the percentages are absolute, that is, they reflect the status of all neurons examined, including the majority that was not labeled for Arc. To aid interpretation, the percent increase in Arc-positive neurons is also given next to the bars for the control mice. Interestingly, there was significant salient context-specific labeling in dCA3 but not dCA1 in the $D b h^{-1-}$ mice, and there was a significant difference between genotypes in dCA1 but not dCA3. In the dDG there was no salient context-specific labeling in either genotype using the standard analysis (Fig. 5) or a second analysis that took into account the extended duration of nuclear labeling.

The ventral hippocampus was also examined because studies suggest that some of its functional roles may differ from the dorsal hippocampus (Kjelstrup et al. 2002; Maren and Holt 2004). Labeling was about half as frequent as that observed in the dorsal hippocampus (Fig. 6). Owing to very sparse labeling and lack of salient context-specific effects in the dDG, the vDG was not quantified. In vCA3 there was no significant salient contextspecific labeling. However, in vCA1 there was significant salient context-specific labeling in control but not $\mathrm{Dbh}^{-1-}$ mice, similar to dCA1.
Salient context-specific labeling in other brain regions Because of the success in using Arc catFISH to identify salient context-specific labeling in the hippocampus, and because of the attractive within-subject design of Arc catFISH, we asked whether salient context-specific activation could be observed in additional brain regions. These regions were either predicted to exhibit salient context-specific activation based on prior functional studies, known to be areas of considerable input or output with respect to the hippocampus, and/or were observed to exhibit obvious salient context-specific activation during the course of the study.

Further analysis began with the amygdala because it is critical for acquisition and expression of conditioned fear (Kim and Fanselow 1992; Helmstetter and Bellgowan 1994; Muller et al. 1997). In the amygdala, there was significant salient contextspecific labeling in all four regions examined in control mice: the lateral, central, basolateral, and basomedial nuclei (Fig. 7). In contrast, only in the lateral nucleus was there significant salient context-specific labeling ( $40 \%$ of control) in $\mathrm{Dbh}^{-1-}$ mice. Furthermore, in the lateral, basomedial, and central nuclei, salient context-specific $A r c$ induction in $D b h^{-1-}$ mice was significantly impaired relative to controls.

In the course of these studies we noted striking salient context-specific labeling throughout the dorsolateral half of the caudate/putamen (dlCPu). For comparison, labeling was also quantified in the dorsomedial $\mathrm{CPu}(\mathrm{dmCPu})$. Significant salient context-specific labeling was present in control and $\mathrm{Dbh}^{-/-}$mice in the dlCPu. However, salient context-specific labeling in the mutants was only $30 \%$ of that for controls, a significant reduction. No salient context-specific labeling was observed in the dmCPu. As for other structures in the striatum, studies suggest that the nucleus accumbens (NAc) plays an important role in contextual fear conditioning (Riedel et al. 1997; Haralambous and Westbrook 1999; Levita et al. 2002). Using Arc catFISH, we found significant salient context-specific labeling in the shell of the
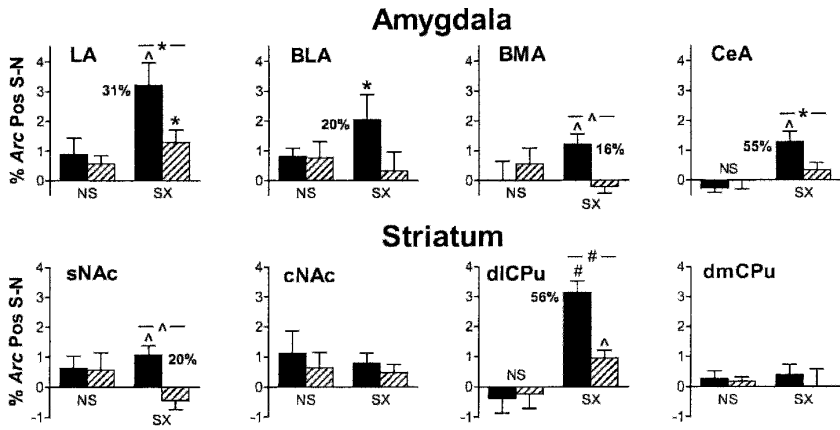

Posterior Cortex
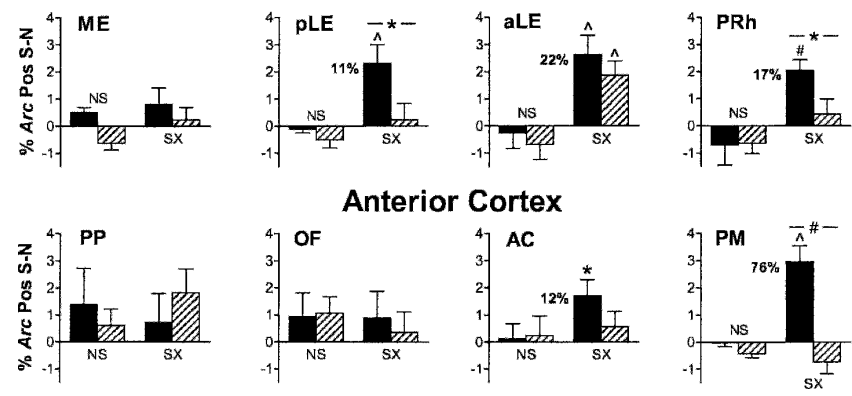

Figure 7. Salient context-specific labeling in additional brain regions. Data are presented as in Figure 6 except that percentage labeling by compartment is not shown. Salient context-specific labeling is observed in several brain regions in controls and in some cases in $D b h^{-1-}$ mice, although only in the aLE is the labeling equivalent to that in controls. 
NAc in control but not $\mathrm{Dbh}^{-1-}$ mice, and a significant difference between genotypes (Fig. 7). In contrast, no salient contextspecific labeling was observed in the core of the NAc.

Labeling in the cortex was also examined. Several parahippocampal cortical regions were studied because of their extensive connections with the hippocampus and their role in mnemonic processes (Eichenbaum 2000; Witter et al. 2000; Brown and Aggleton 2001; Egorov et al. 2002). No salient context-specific labeling was present in the medial entorhinal cortex; however, the posterior lateral entorhinal cortex exhibited significant salient context-specific labeling that was present only in control mice (Fig. 7). More rostrally, there was significant salient contextspecific labeling in both genotypes in the anterior lateral entorhinal cortex, while in the adjacent perirhinal cortex there was significant salient context-specific labeling that was present only in control mice.

In the frontal cortex, we noted striking salient contextspecific labeling in the primary motor cortex. When this was quantified and compared to other regions of the frontal cortex, we found salient context-specific labeling in the primary motor cortex and to a lesser degree in the anterior cingulate cortex that was present only in control mice (Fig. 7). In contrast, there was no salient context-specific activation of the orbitofrontal cortex. Finally, when we examined the posterior parietal sensory association area, no salient context-specific labeling was observed.

\section{Discussion}

\section{Applicability of Arc catFISH}

This study highlights the general applicability of $\operatorname{Arc}$ catFISH for studying neuronal activation. The results are consistent with studies using Arc catFISH in the rat (Guzowski et al. 1999) and extend those observations to the mouse and to many additional brain regions. For interpreting results from Arc catFISH, it is important to stress that induction of Arc is not meant to indicate whether a neuron fired action potentials during a behavioral epoch. In general, the relationship between neural activity and IEG activation is complex (Clayton 2000; Fields et al. 2001). Rather, induction of Arc should be regarded as a marker of "genomic activation" that likely represents a threshold or specific pattern of postsynaptic activity.

For the purpose of studying memory retrieval and its sequelae, we were able to identify brain regions using Arc catFISH that had significantly greater labeling unique to the salient versus the neutral context. Arc was chosen as an IEG marker of neuronal activity because of its previously described utility for within-subjects analysis using catFISH (Guzowski et al. 1999, 2001). Because Arc is implicated in synaptic plasticity and memory formation (Guzowski et al. 2000), it was not clear whether Arc catFISH would identify neurons uniquely activated during memory retrieval. The fact that it did could reflect a role for Arc in reconsolidation following memory retrieval. Our results in control mice are consistent with results from previous imaging studies examining the IEGs Fos or zif268 following retrieval tests for contextual fear conditioning that used brief training protocols (Milanovic et al. 1998; Hall et al. 2001a; Thomas et al. 2002; Frankland et al. 2004).

We observed some double labeling for Arc in all brain regions. This was expected because there were many similarities as well as some important differences between exposure to a neutral and a salient context. For example, in both situations animals in the same room were aroused, picked up by the tail, and placed into a relatively novel context. Significant overlap in neuronal activation could be present because of similar sensorimotor experiences and arousal in both situations. Indeed, it was imperative that this be identified and subtracted in order to detect la- beling due to specific differences between the two situations (e.g., retrieval, fear, freezing).

Critical to the analyses performed in this study on the role of adrenergic signaling, basal levels of Arc, as well as the levels induced by a neutral context, were unaffected in the $D b h^{-1-}$ mice. This is perhaps surprising because lesions of the locus coeruleus, which supplies much of the adrenergic innervation to the forebrain, cause reductions in Arc labeling (Cirelli and Tononi 2000). However, those experiments measured IEG expression following prolonged wakefulness with repeated novel object exposure, whereas our experiments examined short-term induction of $A r c$ in response to a change in context. Another difference of potential importance is that lesions incapacitate adrenergic terminals, whereas the adrenergic terminals are intact (except for the absence of NE/E) in the $D b h^{-1-}$ mice (Jin et al. 2004).

\section{Memory retrieval, the hippocampus, and adrenergic signaling}

Using Arc catFISH, we examined how NE might act to promote contextual memory retrieval in the hippocampus. We focused on neuronal activation in the hippocampus during retrieval because infusion of $\beta_{1}$-adrenergic receptor antagonists into the dorsal hippocampus selectively impairs contextual fear memory retrieval, as does systemic NE/E deficiency in $\mathrm{Dbh}^{-/-}$mice (Murchison et al. 2004). In this and the previous study, freezing to the training context is reduced but not eliminated in the mutant mice and in control mice treated with a $\beta_{1}$ antagonist. There are several potential explanations for this. For example, it could be that retrieval is impaired but not eliminated because factors in addition to NE promote some retrieval in the hippocampus. Residual freezing could also be present in response to elements contained within the context (cued fear), which is normal in the mutant mice (Murchison et al. 2004).

Importantly, neither blocking $\beta_{1}$ receptors in the dorsal hippocampus nor systemic deficiency of NE/E alters fear in general as assessed by freezing in response to the training tone $1 \mathrm{~d}$ after conditioning or in response to the training context $1 \mathrm{wk}$ after conditioning (Murchison et al. 2004). It was our hypothesis then that in the absence of NE/E, one or more subfields of the hippocampus would exhibit impaired activation during contextual memory retrieval. Our results indicate that CA3 is activated normally in the absence of NE/E. In contrast, CA1 exhibits salient context-specific activation that is significantly and considerably reduced in the absence of NE/E.

One consideration is whether the increased labeling for Arc in CA1 following exposure to the salient context is due to arousal, fear, or freezing, for example, rather than memory retrieval. Importantly, results from several studies demonstrate minimal or no change in Fos or zif268 levels in CA1 when testing cued fear conditioning after habituation to the context, or when testing contextual fear conditioning $\sim 1$ mo after training (Hall et al. 2001a,b; Frankland et al. 2004). Under those conditions subjects exhibited high levels of freezing (fear) to the context or cue without induction of Fos or zif268 in CA1, dissociating IEG expression and arousal, fear, and freezing behavior.

One could argue that induction of Arc reflects processes that are distinct from those reflected by induction of Fos or zif268. For example, even though adrenergic signaling in the hippocampus mediates contextual memory retrieval, salient context-specific labeling for Arc (but not Fos or zif268) in the hippocampus could reflect increased arousal, fear, or freezing that results from fear memory retrieval. However, it is interesting to note that results examining induction of Fos in the hippocampus following contextual fear memory retrieval in control and $\mathrm{Dbh}^{-1-}$ mice (Tho- 
mas et al. 2004) mirror those for the induction of Arc presented here. Furthermore, a general deficit in arousal in the $D b h^{-1-}$ mice that would impinge upon induction of Arc might have been manifest when mice were placed in the neutral context (arousing because it is relatively novel). A large majority of the Arc induction observed in the salient context was also observed in the neutral context. However, such induction was entirely normal in the $\mathrm{Dbh}^{-1-}$ mice. Of course, it is possible that the magnitude or pattern of activation induced by arousal in a relatively novel, but neutral, context differs from that for arousal in a salient context.

The patterns of salient context-specific Arc induction that we have observed in the hippocampus are interesting to consider in light of models suggesting CA3 is important for contextual memory storage and retrieval, while CA1 is important for comparing memory of context coming from CA3 to the current context coming from the perforant path directly to CA1 (Treves and Rolls 1994; Lisman 1999). Based on these models, we hypothesize that perforant path activity to the hippocampus is normal in the absence of NE, allowing normal contextual memory storage in CA3 and normal online contextual information processing in CA1. This hypothesis is consistent with the normal activation of CA1 in a neutral context and normal activation of CA3 in the neutral and salient contexts in the $\mathrm{Dbh}^{-1-}$ mice. The model and results suggest that activation of $\mathrm{CA} 3$ during contextual memory retrieval fails to be properly transmitted to CA1. This could be for several reasons. $\beta_{1}$-adrenergic receptors, which are critical for the actions of NE in promoting memory retrieval, are expressed primarily by CA1 pyramidal neurons and dentate granule cells (Nicholas et al. 1993). Thus, it is possible that $\beta_{1}$-adrenergic receptors expressed by CA1 pyramidal neurons play a key role in gating the strength of the Schäffer collateral input from CA3. Alternatively or in addition, $\beta_{1}$-adrenergic receptors expressed by DG cells could indirectly gate the firing of CA3 pyramidal neurons via the mossy fiber pathway. The latter seems less likely given the normal induction of $A r c$ in dCA3 in the $\mathrm{Dbh}^{-1-}$ mice during retrieval. However, because the relationship between induction of IEGs and neuronal firing is complex (Clayton 2000; Fields et al. 2001), it is possible that the synaptic activation that leads to normal Arc induction does not lead to appropriate firing in CA3.

\section{Salient context-specific Arc labeling in other brain regions}

We predicted that when retrieval was impaired, activation in other brain regions would also be altered. This is because impaired retrieval leads to reduced fear and freezing. In the amygdala, salient context-specific labeling was present in all four nuclei examined in control mice, consistent with a requirement for each of these in fear conditioning (Goosens and Maren 2001). In contrast, in $\mathrm{Dbh}^{-1-}$ mice salient context-specific labeling was present only in the lateral nucleus, where there was significantly less labeling than in controls.

In the striatum, salient context-specific labeling in the dl$\mathrm{CPu}$ was striking, both because it represented $\sim 30 \%$ of total ArC labeling in this region, and because it was anatomically extensive (dorsal to ventral and rostral to caudal). Interestingly, the dlCPu receives cortical input largely from the somatosensory and motor cortices, whereas the medial $\mathrm{CPu}$ receives input from the visual, auditory, and olfactory cortices (McGeorge and Faull 1989). Because salient context-specific labeling was significantly reduced in the $\mathrm{Dbh}^{-1-}$ mice in this region, it seems reasonable to hypothesize that the dlCPu is important for mediating behavioral output, for example, freezing. This is consistent with there being salient context-specific labeling throughout the dlCPu because freezing entails fixation of posture (motor inhibition) across the somatic axis. The primary motor region of the prefrontal cortex exhibited a similar pattern of labeling to that for the dlCPu, suggesting a potential role in mediating freezing as well.

The parahippocampal cortices (perirhinal, postrhinal, and entorhinal) are multimodal association areas that process and relay sensory information between the hippocampus and other cortical regions (Insausti et al. 1997; Burwell and Amaral 1998; Dolorfo and Amaral 1998; Naber and Witter 1998; Witter et al. 2000). Lesion studies of the entorhinal or perirhinal cortex suggest that these regions may contribute to contextual fear conditioning (Corodimas and LeDoux 1995; Phillips and LeDoux 1995; Maren and Fanselow 1997). We observed varying degrees of salient context-specific activation that depended on the area examined. Interestingly, the anterior lateral entorhinal cortex exhibited salient context-specific activation, but it was not dependent on NE/E, similar to what was observed in CA3. This pattern of activity suggests that this region may process information either en route to the hippocampus (e.g., upstream of DG/ CA3) or independently of the hippocampus. Two of the regions (posterior lateral entorhinal and anterior perirhinal) exhibited salient context-specific activation that was not present in the absence of NE/E. These regions may subserve contextual information processing downstream of the hippocampus because the labeling was dependent on NE/E and was present in the deep layers of these regions, areas that receive much of the cortical hippocampal output via CA1 and the subiculum.

\section{Conclusions}

This study demonstrates that Arc catFISH can be used in mice to map activity in many regions of the brain during distinct behavioral epochs. Within the hippocampus, NE is required for normal activation of CA1 but not CA3 during retrieval of a salient contextual memory. Because normal behavior is restored in $\mathrm{Dbh}^{-1-}$ mice when testing contextual fear following infusion of a $\beta$-adrenergic receptor agonist into the dorsal hippocampus (Murchison et al. 2004), we hypothesize that the lack of NE-dependent retrieval in the hippocampus in untreated $\mathrm{Dbh}^{-/-}$mice results in reduced salient context-specific activation of multiple "downstream" brain regions (e.g., the amygdala, dlCPu, and some cortical areas) important for mediating appropriate, contextdependent behavior. This could be investigated in future studies by examining IEG induction following context exposure after infusing a $\beta_{1}$-adrenergic receptor antagonist into the dorsal hippocampus.

In addition, it would be interesting to map contextual memory retrieval in even greater detail in future studies. For example, one could compare salient context-specific activation in proximal versus distal regions (along the transverse axis) of CA1 and the subiculum because anatomic studies indicate distinct differences in connectivity within these regions and in their extrahippocampal projections (Naber et al. 2000; Witter et al. 2000). A clearer picture of activation within the parahippocampal cortices could be obtained by analyzing many more areas and their various layers. Extensive analyses such as these using catFISH would be greatly aided by automated methods for quantifying labeled neurons that are now under development (Lin et al. 2003).

\section{Materials and Methods}

\section{Animals and behavior}

$D b h^{-1-}$ mice were rescued prenatally and housed as described (Ouyang et al. 2004). Studies were in accordance with NIH guidelines and had the approval of IACUC at the University of Pennsylvania. Sex-matched littermate $D b h^{+/-}$mice (4-5 mo old) were used as controls because they have normal tissue levels of NE/E 
Table 1. Approximate mean total number of cells analyzed per mouse by brain region

\begin{tabular}{|c|c|c|c|c|c|c|c|c|c|c|c|c|c|c|c|c|c|c|c|}
\hline \multicolumn{4}{|c|}{ Hippocampus } & \multicolumn{4}{|c|}{ Amygdala } & \multicolumn{4}{|c|}{ Striatum } & \multicolumn{8}{|c|}{ Cortex } \\
\hline dCA3 & dCA1 & vCA3 & vCA1 & LA & BLA & BMA & $\mathrm{CeA}$ & sNAc & cNAc & dICPu & $\mathrm{dmCPu}$ & ME & pLE & aLE & PRh & PP & OF & AC & PM \\
\hline 240 & 320 & 340 & 380 & 440 & 400 & 380 & 560 & 640 & 720 & 480 & 720 & 520 & 360 & 300 & 380 & 520 & 540 & 480 & 320 \\
\hline
\end{tabular}

See Figure 1 for anatomic abbreviations and locations.

and are phenotypically indistinguishable from $\mathrm{Dbh}^{+/+}$mice (Thomas et al. 1998). Four mice of each genotype were used for each treatment group.

Fear conditioning was performed as described in context $\mathrm{S}$ (ENV-010MC; Med Associates), which was a clear Plexiglas box with a metal bar floor $20 \times 16 \mathrm{~cm}$ and $21 \mathrm{~cm}$ tall (Murchison et al. 2004). On day 1 , following $2 \mathrm{~d}$ of handling, mice were placed for 3 min in context $\mathrm{N}$, which was a Plexiglas cylinder with a green wire grid floor, $21 \mathrm{~cm}$ diameter, $24 \mathrm{~cm}$ tall, having vertical white and green stripes around two-thirds of the wall and olfactory cues distinct from context S. About $3 \mathrm{~h}$ later they were placed in context $S$ (same room) for $3 \mathrm{~min}$ (see Fig. 3A). At 2 min, a tone was activated for $30 \mathrm{sec}$ and a 2 -sec, 1-mA footshock coterminated with the tone for mice in all groups except the no shock (NS) group. For basal Arc expression, mice trained on day 1 were removed from the home cage (group HC) on day 2. For analysis of the time course of Arc expression, mice were sacrificed at $5,15,20,30$, or $35 \mathrm{~min}$ after exposure to context $\mathrm{S}$ for $3 \mathrm{~min}$ on day 2 . For analysis of memory retrieval, exposure to contexts $\mathrm{S}$ and $\mathrm{N}$ was performed on day 2 for 3 min each without shock. There was a 20-min interval in the home cage between contexts $\mathrm{S}$ and $\mathrm{N}$. Percent freezing was estimated by scoring the presence or absence of nonrespiratory movement every 5 sec. Only mice that were resting before context testing or death (group HC) were used.

\section{catFISH}

Mice were sacrificed on day 2 after being removed from their home cage or $5 \mathrm{~min}$ following the second context exposure. Their brains were rapidly dissected and stored $\left(-80^{\circ} \mathrm{C}\right)$. Six brains (at least one from each group) were mounted together, and coronal sections $(20 \mu \mathrm{m})$ were cut by cryostat (HM505E; Microm). Sections were thaw-mounted onto slides and stored $\left(-80^{\circ} \mathrm{C}\right)$. Digoxigenin-labeled antisense and sense Arc riboprobes $(\sim 3 \mathrm{~kb})$ were prepared from linearized mouse cDNA (P. Worley, Johns Hopkins University, Baltimore, MD) using a kit (Roche). FISH was performed as described (Guzowski et al. 1999) using antidigoxigenin-horseradish peroxidase (Roche) and TSA-Direct fluorescence amplification (PerkinElmer). Slides were coverslipped with anti-fade medium containing DAPI (Vectashield; Vector Laboratories) and sealed. Sections were viewed using epifluorescence (Eclipse E600W; Nikon). To detect FITC, 494-nm excitation and 520-nm emission wavelengths were used. To detect DAPI, 360-nm excitation and 456-nm emission wavelengths were used. Images $(1280 \times 1024$ pixels $)$ were acquired using a monochrome CCD camera (ORCA-100; Hamamatsu Photonics). RGB TIFF images were recorded using Image-Pro Plus (Media Cybernetics). For compartment analysis, the entire field was analyzed $(80 \times$ magnification, $106 \times 85 \mu \mathrm{m}, 83 \mathrm{~nm} /$ pixel for CA3 and CA1; $40 \times$ magnification, $212 \times 170 \mu \mathrm{m}, 83 \mathrm{~nm} /$ pixel for other regions). In all, 20 images $(1 \mu \mathrm{m})$ were recorded along the $z$-axis of each section, and Autodeblur software (AutoQuant Imaging) was used for sharpening. Small, bright, uniformly DAPIstained nuclei (from putative glial cells) were not analyzed. All other whole nuclei were analyzed from top to bottom. Nucleuspositive $(\mathrm{Nu})$ neurons had two small, intense intranuclear fluorescent foci; cytoplasm-positive (Cy) neurons contained perinuclear/cytoplasmic labeling in multiple optical sections. Four adjacent sections were analyzed bilaterally for each brain region from each mouse. Approximate mean total numbers of cells analyzed per mouse for each brain region are given in Table 1 . There were a total of 32 mice used for catFISH ( 4 mice $\times 2$ genotypes $\times 4$ conditions). The total number of cells analyzed for each of the 20 regions besides the DG ranged from $\sim 8000$ to $\sim 23,000$. For the DG, there were estimated to be $>300$ cells/field; thus, the number of positive cells/field is reported because negative cells were not scored owing to sparse labeling under all conditions.

\section{Statistical analysis}

Data were analyzed with Statistica 6.0 (StatSoft) using factorial two-way ANOVA of genotype and condition. Depending on the experiment, condition was either "context" for behavioral data, "treatment group" for total Arc labeling, or "type of labeling" for Arc compartment analysis. Post hoc comparisons were made using Duncan's range test. For analysis of context-specific labeling, each animal served as its own control for catFISH because of the within-subject design. Therefore, the difference for labeling between contexts (neutral - neutral or salient - neutral) was calculated for each animal. These were averaged to obtain summary data for each group. The null hypothesis was that the mean residual values would not be significantly different from zero. This was our planned comparison and was tested using a one-sample $t$-test. Our one additional planned comparison was between genotypes within the same group, which was made using a Student's unpaired $t$-test. Because these two planned comparisons were on related rather than independent data, no adjustment was made for $\alpha<0.05$. Levels of significance are indicated as: *, $P<0.05 ; \wedge, P<0.01 ; \#, P<0.001$.

\section{Acknowledgments}

This work was supported by NIMH (MH063352 to S.A.T. and MH060123 to J.F.G.). We thank H. Luo for technical assistance, P. Worley for the Arc cDNA, and Sumitomo Pharmaceuticals for their generous gift of L-DOPS.

\section{References}

Brown, M.W. and Aggleton, J.P. 2001. Recognition memory: What are the roles of the perirhinal cortex and hippocampus? Nat. Rev. Neurosci. 2: 51-61.

Burwell, R.D. and Amaral, D.G. 1998. Cortical afferents of the perirhinal, postrhinal, and entorhinal cortices of the rat. J. Comp. Neurol. 398: 179-205.

Cirelli, C. and Tononi, G. 2000. Differential expression of plasticity-related genes in waking and sleep and their regulation by the noradrenergic system. J. Neurosci. 20: 9187-9194.

Clayton, D.F. 2000. The genomic action potential. Neurobiol. Learn. Mem. 74: $185-216$.

Corodimas, K.P. and LeDoux, J.E. 1995. Disruptive effects of posttraining perirhinal cortex lesions on conditioned fear: Contributions of contextual cues. Behav. Neurosci. 109: 613-619.

Dolorfo, C.L. and Amaral, D.G. 1998. Entorhinal cortex of the rat: Topographic organization of the cells of origin of the perforant path projection to the dentate gyrus. J. Comp. Neurol. 398: 25-48.

Egorov, A.V., Hamam, B.N., Fransen, E., Hasselmo, M.E., and Alonso, A.A. 2002. Graded persistent activity in entorhinal cortex neurons. Nature 420: $173-178$.

Eichenbaum, H. 2000. A cortical-hippocampal system for declarative memory. Nat. Rev. Neurosci. 1: 41-50.

Fanselow, M.S. and LeDoux, J.E. 1999. Why we think plasticity underlying Pavlovian fear conditioning occurs in the basolateral amygdala. Neuron 23: 229-232.

Fields, R.D., Eshete, F., Dudek, S., Ozsarac, N., and Stevens, B. 2001. Regulation of gene expression by action potentials: Dependence on complexity in cellular information processing. Novartis Found. Symp. 239: 160-172; discussion 172-176, 234-240.

Frankland, P.W., Bontempi, B., Talton, L.E., Kaczmarek, L., and Silva, A.J. 2004. The involvement of the anterior cingulate cortex in 
remote contextual fear memory. Science 304: $881-883$.

Goosens, K.A. and Maren, S. 2001. Contextual and auditory fear conditioning are mediated by the lateral, basal, and central amygdaloid nuclei in rats. Learn. Mem. 8: 148-155.

Guzowski, J.F., McNaughton, B.L., Barnes, C.A., and Worley, P.F. 1999. Environment-specific expression of the immediate-early gene Arc in hippocampal neuronal ensembles. Nat. Neurosci. 2: 1120-1124.

Guzowski, J.F., Lyford, G.L., Stevenson, G.D., Houston, F.P., McGaugh, J.L., Worley, P.F., and Barnes, C.A. 2000. Inhibition of activity-dependent arc protein expression in the rat hippocampus impairs the maintenance of long-term potentiation and the consolidation of long-term memory. J. Neurosci. 20: 3993-4001.

Guzowski, J.F., McNaughton, B.L., Barnes, C.A., and Worley, P.F. 2001. Imaging neural activity with temporal and cellular resolution using FISH. Curr. Opin. Neurobiol. 11: 579-584.

Hall, J., Thomas, K.L., and Everitt, B.J. 2001a. Cellular imaging of zif268 expression in the hippocampus and amygdala during contextual and cued fear memory retrieval: Selective activation of hippocampal CA1 neurons during the recall of contextual memories. J. Neurosci. 21: $2186-2193$.

. 2001b. Fear memory retrieval induces CREB phosphorylation and Fos expression within the amygdala. Eur. J. Neurosci. 13: $1453-1458$.

Haralambous, T. and Westbrook, R.F. 1999. An infusion of bupivacaine into the nucleus accumbens disrupts the acquisition but not the expression of contextual fear conditioning. Behav. Neurosci. 113: $925-940$

Helmstetter, F.J. and Bellgowan, P.S. 1994. Effects of muscimol applied to the basolateral amygdala on acquisition and expression of contextual fear conditioning in rats. Behav. Neurosci. 108: $1005-1009$.

Hof, P.R., Young, W.G., Bloom, F.E., Belichenko, P.V., and Celio, M.R. 2000. Comparative cytoarchitectonic atlas of the C57BL/6 and $129 / \mathrm{Sv}$ mouse brains. Elsevier Science B.V., Amsterdam.

Holt, W. and Maren, S. 1999. Muscimol inactivation of the dorsal hippocampus impairs contextual retrieval of fear memory. $J$. Neurosci. 19: 9054-9062.

Insausti, R., Herrero, M.T., and Witter, M.P. 1997. Entorhinal cortex of the rat: Cytoarchitectonic subdivisions and the origin and distribution of cortical efferents. Hippocampus 7: 146-183.

Jin, S.-H., Kim, H.J.T., Harris, D.C., and Thomas, S.A. 2004. Postnatal development of the cerebellum and adrenergic system is independent of norepinephrine and epinephrine. J. Comp. Neurol. 477: 300-309.

Kim, J.J. and Fanselow, M.S. 1992. Modality-specific retrograde amnesia of fear. Science 256: 675-677.

Kim, J.J., Fanselow, M.S., DeCola, J.P., and Landeira-Fernandez, J. 1992. Selective impairment of long-term but not short-term conditional fear by the N-methyl-D-aspartate antagonist APV. Behav. Neurosci. 106: $591-596$.

Kjelstrup, K.G., Tuvnes, F.A., Steffenach, H.A., Murison, R., Moser, E.I., and Moser, M.B. 2002. Reduced fear expression after lesions of the ventral hippocampus. Proc. Natl. Acad. Sci. 99: 10825-10830.

Kubie, J.L. and Ranck, J.B.J. 1983. Sensory-behavioral correlates in individual hippocampal neurons in three situations: Space and context. In Neurobiology of the hippocampus (ed. W. Seifert), pp. 433-447. Academic Press, New York.

Levita, L., Dalley, J.W., and Robbins, T.W. 2002. Disruption of Pavlovian contextual conditioning by excitotoxic lesions of the nucleus accumbens core. Behav. Neurosci. 116: 539-552.

Lin, G., Adiga, U., Olson, K., Guzowski, J.F., Barnes, C.A., and Roysam, B. 2003. A hybrid 3D watershed algorithm incorporating gradient cues and object models for automatic segmentation of nuclei in confocal image stacks. Cytometry 56A: 23-36.

Link, W., Konietzko, U., Kauselmann, G., Krug, M., Schwanke, B., Frey, U., and Kuhl, D. 1995. Somatodendritic expression of an immediate early gene is regulated by synaptic activity. Proc. Natl. Acad. Sci. 92: 5734-5738.

Lisman, J.E. 1999. Relating hippocampal circuitry to function: Recall of memory sequences by reciprocal dentate-CA3 interactions. Neuron 22: $233-242$.

Lyford, G.L., Yamagata, K., Kaufmann, W.E., Barnes, C.A., Sanders, L.K., Copeland, N.G., Gilbert, D.J., Jenkins, N.A., Lanahan, A.A., and Worley, P.F. 1995. Arc, a growth factor and activity-regulated gene, encodes a novel cytoskeleton-associated protein that is enriched in neuronal dendrites. Neuron 14: 433-445.

Maren, S. and Fanselow, M.S. 1997. Electrolytic lesions of the fimbria/fornix, dorsal hippocampus, or entorhinal cortex produce anterograde deficits in contextual fear conditioning in rats. Neurobiol. Learn. Mem. 67: 142-149.

Maren, S. and Holt, W.G. 2004. Hippocampus and Pavlovian fear conditioning in rats: Muscimol infusions into the ventral, but not dorsal, hippocampus impair the acquisition of conditional freezing to an auditory conditional stimulus. Behav. Neurosci. 118: 97-110.

Mayes, A.R. and Montaldi, D. 2001. Exploring the neural bases of episodic and semantic memory: The role of structural and functional neuroimaging. Neurosci. Biobehav. Rev. 25: 555-573.

McGeorge, A.J. and Faull, R.L. 1989. The organization of the projection from the cerebral cortex to the striatum in the rat. Neuroscience 29: $503-537$.

Milanovic, S., Radulovic, J., Laban, O., Stiedl, O., Henn, F., and Spiess, J. 1998. Production of the Fos protein after contextual fear conditioning of C57BL/6N mice. Brain Res. 784: 37-47.

Muller, J., Corodimas, K.P., Fridel, Z., and LeDoux, J.E. 1997. Functional inactivation of the lateral and basal nuclei of the amygdala by muscimol infusion prevents fear conditioning to an explicit conditioned stimulus and to contextual stimuli. Behav. Neurosci. 111: $683-691$

Murchison, C.F., Zhang, X.-Y., Zhang, W.-P., Ouyang, M., Lee, A., and Thomas, S.A. 2004. A distinct role for norepinephrine in memory retrieval. Cell 117: 131-143.

Myers, K.M. and Davis, M. 2002. Behavioral and neural analysis of extinction. Neuron 36: 567-584.

Naber, P.A. and Witter, M.P. 1998. Subicular efferents are organized mostly as parallel projections: A double-labeling, retrograde-tracing study in the rat. J. Comp. Neurol. 393: 284-297.

Naber, P.A., Witter, M.P., and Lopes Silva, F.H. 2000. Networks of the hippocampal memory system of the rat. The pivotal role of the subiculum. Ann. NY Acad. Sci. 911: 392-403.

Nader, K., Schafe, G.E., and LeDoux, J.E. 2000. The labile nature of consolidation theory. Nat. Rev. Neurosci. 1: 216-219.

Nicholas, A.P., Pieribone, V.A., and Hokfelt, T. 1993. Cellular localization of messenger RNA for $\beta-1$ and $\beta-2$ adrenergic receptors in rat brain: An in situ hybridization study. Neuroscience 56: 1023-1039.

Ouyang, M., Hellman, K., Abel, T., and Thomas, S.A. 2004. Adrenergic signaling plays a critical role in the maintenance of waking and in the regulation of REM sleep. J. Neurophysiol. 92: 2071-2082.

Phillips, R.G. and LeDoux, J.E. 1992. Differential contribution of amygdala and hippocampus to cued and contextual fear conditioning. Behav. Neurosci. 106: 274-285.

. 1995. Lesions of the fornix but not the entorhinal or perirhinal cortex interfere with contextual fear conditioning. J. Neurosci. 15: $5308-5315$.

Riedel, G., Harrington, N.R., Hall, G., and Macphail, E.M. 1997. Nucleus accumbens lesions impair context, but not cue, conditioning in rats. Neuroreport 8: 2477-2481.

Schacter, D.L. and Wagner, A.D. 1999. Medial temporal lobe activations in fMRI and PET studies of episodic encoding and retrieval. Hippocampus 9: 7-24.

Steward, O., Wallace, C.S., Lyford, G.L., and Worley, P.F. 1998. Synaptic activation causes the mRNA for the IEG Arc to localize selectively near activated postsynaptic sites on dendrites. Neuron 21: 741-751.

Thomas, S.A., Matsumoto, A.M., and Palmiter, R.D. 1995. Noradrenaline is essential for mouse fetal development. Nature 374: 643-646.

Thomas, S.A., Marck, B.T., Palmiter, R.D., and Matsumoto, A.M. 1998. Restoration of norepinephrine and reversal of phenotypes in mice lacking dopamine $\beta$-hydroxylase. J. Neurochem. 70: 2468-2476.

Thomas, K.L., Hall, J., and Everitt, B.J. 2002. Cellular imaging with zif 268 expression in the rat nucleus accumbens and frontal cortex further dissociates the neural pathways activated following the retrieval of contextual and cued fear memory. Eur. J. Neurosci. 16: $1789-1796$.

Thomas, S.A., Zhang, W.-P., Murchison, C.F., and Guzowski, J.F. 2004. Mapping contextual memory retrieval and its dependence on norepinephrine. Soc. Neurosci. Abstracts 30: No. 1008-1011.

Thompson, L.T. and Best, P.J. 1990. Long-term stability of the place-field activity of single units recorded from the dorsal hippocampus of freely behaving rats. Brain Res. 509: 299-308.

Treves, A. and Rolls, E.T. 1994. Computational analysis of the role of the hippocampus in memory. Hippocampus 4: 374-391.

Vazdarjanova, A.I., Houston, F.P., Worley, P., Barnes, C.A., and Guzowski, J.F. 2002. Similar activity dependent immediate-early gene transcriptional responses in hippocampal pyramidal neurons and dentate gyrus granule cells. Soc. Neurosci. Abstracts 28: No. 678.672 .

Wilson, M.A. and McNaughton, B.L. 1993. Dynamics of the hippocampal ensemble code for space. Science 261: 1055-1058.

Witter, M.P., Naber, P.A., van Haeften, T., Machielsen, W.C., Rombouts, S.A., Barkhof, F., Scheltens, P., and Lopes da Silva, F.H. 2000. Cortico-hippocampal communication by way of parallel parahippocampal-subicular pathways. Hippocampus 10: 398-410.

Received September 17, 2004; accepted in revised form March 22, 2005. 


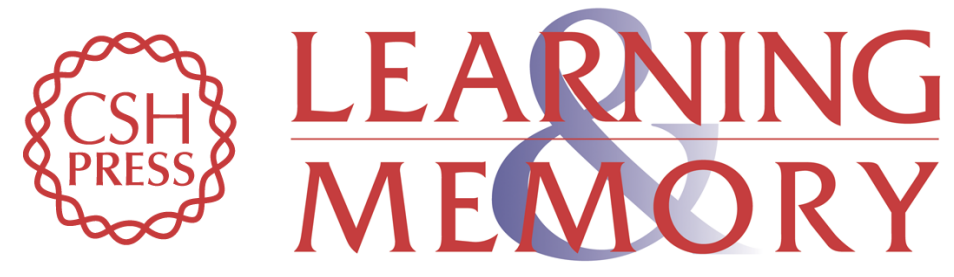

\section{Mapping neuronal activation and the influence of adrenergic signaling during contextual memory retrieval}

Wei-Ping Zhang, John F. Guzowski and Steven A. Thomas

Learn. Mem. 2005, 12:

Access the most recent version at doi:10.1101/lm.90005

References This article cites 55 articles, 11 of which can be accessed free at: http://learnmem.cshlp.org/content/12/3/239.full.html\#ref-list-1

License

Email Alerting Receive free email alerts when new articles cite this article - sign up in the box at the Service top right corner of the article or click here. 